\title{
Fundamentos e análise de tintas refratárias de alta emissividade
}

\section{(Fundamentals and analysis of high emissivity refractory coatings)}

\author{
A. A. C. Mourão, D. O. Vivaldini, V. R. Salvini, V. C. Pandolfelli \\ Grupo de Engenharia de Microestrutura de Materiais, Departamento de Engenharia de Materiais, Universidade \\ Federal de S.Carlos, Rod. Washington Luiz km 235, S. Carlos, SP, Brasil 13565-905
}

\begin{abstract}
Resumo
O presente trabalho buscou analisar patentes produzidas pela NASA e estudos correlatos na área de tintas refratárias de alta emissividade, segundo os princípios que regem as propriedades termo-ópticas dos materiais, as quais compreendem: absorção, refletividade, transmissão e emissão de radiação eletromagnética. Primeiramente, visou-se a compreensão destes fenômenos físicos e as leis que os governam. Posteriormente, buscou-se aplicá-los destacando e explicando a evolução observada na composição das tintas de alta emissividade utilizadas em veículos espaciais. Por fim, compreendeu-se que o índice de absorção dos materiais é decorrente do modo como estes interagem com a energia radiante incidente. Além disso, constatou-se que a emissividade dos materiais utilizados está associada principalmente ao balanço entre o índice de absorção e a refletividade. Tal estudo e acompanhamento do desenvolvimento tecnológico das tintas refratárias de alta emissividade permitem uma compreensão mais profunda sobre o seu mecanismo de atuação e potencial uso em outros sistemas, como por exemplo nos fornos industriais revestidos com material refratário que operam a altas temperaturas.
\end{abstract}

Palavras-chave: tintas refratárias, emissividade.

\begin{abstract}
The objective of the present paper is to analyze the patents produced by NASA and other related studies in the area of high emissivity refractory coatings, according to the fundamentals that rule the thermo-optical materials properties, which include: absorption, reflectivity, transmission and emission of electromagnetic radiation. Firstly, it aimed to understand these phenomena and their physical laws. Later, those concepts were applied to explain the trend observed in coatings of high emissivity used by NASA. Finally, it was understood that the material absorption index is determined by the way it interacts with the incident radiant energy. Furthermore, it was attested that the emissivity of the materials used are mainly associated to the balance between the absorption index and the reflectivity. This study and understanding of the technological evolution of refractory high emissivity coatings allow a deeper understanding of how they operate and their potential use in other systems, such as industrial furnaces lined with refractory material operating at high temperatures.
\end{abstract}

Keywords: refractory coatings, emissivity.

\section{INTRODUÇÃO}

Na busca para aperfeiçoar o sistema de proteção térmica dos veículos espaciais, a NASA nas últimas décadas desenvolveu e buscou novos compostos para a fabricação de tintas de alta emissividade [1-3], as quais têm por objetivo a proteção da fuselagem por meio da emissão de calor na forma de radiação [4]. Isto se faz necessário em virtude das elevadas temperaturas envolvidas na reentrada na atmosfera terrestre, que podem alcançar $1650^{\circ} \mathrm{C}$. Logo, é de extrema importância o controle da temperatura, uma vez que estas são suficientemente elevadas para fundir a carcaça metálica existente e assim causar acidentes como o ocorrido com o ônibus espacial Columbia [5]. Além disso, em decorrência da atual tendência de redução dos gastos em virtude da elevação do preço da energia elétrica e dos combustíveis $[6,7]$, novas formas de geração e conservação de energia vêm ganhando grande destaque [8] como forma de conter os crescentes gastos com produção [6] e aumentar a competitividade das empresas.
Dentre as inovações que visam à conservação de energia térmica, as tintas de alta emissividade estão sendo cada vez mais utilizadas como uma forma de aumentar a eficiência do isolamento térmico dos fornos industriais que operam a altas temperaturas [9]. Isto se deve a capacidade destas em evitar a perda de calor, ou seja, agir como uma barreira que dificulta a saída da energia presente no sistema.

Entretanto, diferente dos tijolos isolantes, as tintas de alta emissividade não se baseiam na busca de uma baixa condutividade térmica para impedir a transferência de calor, visto que alguns dos seus componentes mais comuns apresentam uma elevada condutividade [1], como o carbeto de silício [10] ou o óxido de ferro [11]. Assim, busca-se nestes materiais uma elevada emissividade, ou seja, uma elevada capacidade de conversão de calor em radiação térmica.

No caso industrial, como nos fornos de fusão ou para queima de material cerâmico, não apenas a emissividade é um fator importante, mas a refletividade da radiação incidente também passa a ser um fator considerável. Isto é 
devido à refletividade também atuar como um mecanismo que dificulta a saída da radiação do sistema, ou seja, sua dispersão através dos refratários.

Pode-se dizer que na prática as tintas de alta emissividade utilizadas no meio industrial têm como objetivo fazer com que parte da energia que seria perdida para o ambiente seja retida conservando o sistema na sua temperatura de trabalho pelo máximo tempo possível. Portanto, tais tintas consistem numa barreira térmica com diferentes aplicações, podendo atuar como um mecanismo para reemitir energia térmica, como ocorre nos ônibus espaciais possibilitando sua reentrada, ou numa forma de evitar a sua perda, o que resulta num menor consumo de combustíveis, como se observa na indústria. Desta forma, pelo estudo das propriedades termoópticas dos materiais, no que se refere à absorção, reflexão, transmissão e emissão de ondas eletromagnéticas, é possível selecionar compostos mais eficientes para a produção destes pigmentos e, assim, entender os critérios de seleção ao desenvolver tintas de alta emissividade. Adicionalmente, estes mesmos princípios podem ser transportados e aplicados para o desenvolvimento e aperfeiçoamento das tintas de alta emissividade utilizadas na indústria, pois seus fundamentos e princípios são os mesmos das tintas usadas em veículos espaciais.

\section{Revisão das propriedades termo-ópticas dos materiais}

Tendo-se em vista o princípio de conservação de energia, existem apenas três possibilidades de interação para uma onda eletromagnética que incide sobre uma superfície. Ela pode ser absorvida (A) pelo meio, refletida (R) ou transmitida (T), de maneira que a soma das frações relativas a cada um desses processos deve ser igual aum, como pode ser observado na equação abaixo [12].

$$
\mathrm{A}+\mathrm{R}+\mathrm{T}=1
$$

\subsection{Absorção e seus mecanismos}

A lei de Beer-Lambert correlaciona à fração de luz transmitida $\left(\mathrm{I} / \mathrm{I}_{0}\right)$ por um meio com certa refletividade $(\mathrm{R})$, coeficiente de extinção $\left(\beta_{\text {ext }}\right)$ e espessura (l), ou seja, estima à atenuação da energia incidente $\left(\mathrm{I}_{0}\right)$ ao passar pelo material segundo a equação B [12].

$$
\mathrm{T}=\mathrm{I} / \mathrm{I}_{0}=(1-\mathrm{R})^{2} \cdot \exp \left(-\beta_{\text {ext }} \cdot 1\right)
$$

O coeficiente $\beta_{\text {ext }}\left(\mathrm{m}^{-1}\right)$ é função da soma dos eventos de absorção e espalhamento que ocorrem no interior do material, consequentemente, para uma matriz homogênea e isenta de defeitos $\beta_{\text {ext }}$, se reduz ao coeficiente de absorção $\left(\beta_{\mathrm{a}}\right)$, o qual é calculado a partir do índice de absorção (k) do material, que é adimensional e corresponde à parte imaginária do índice de refração complexo (n'),como apresentado abaixo.

$$
\beta_{\mathrm{ext}}=\beta_{\mathrm{a}}=\frac{4 . \pi \cdot \kappa}{\lambda}
$$

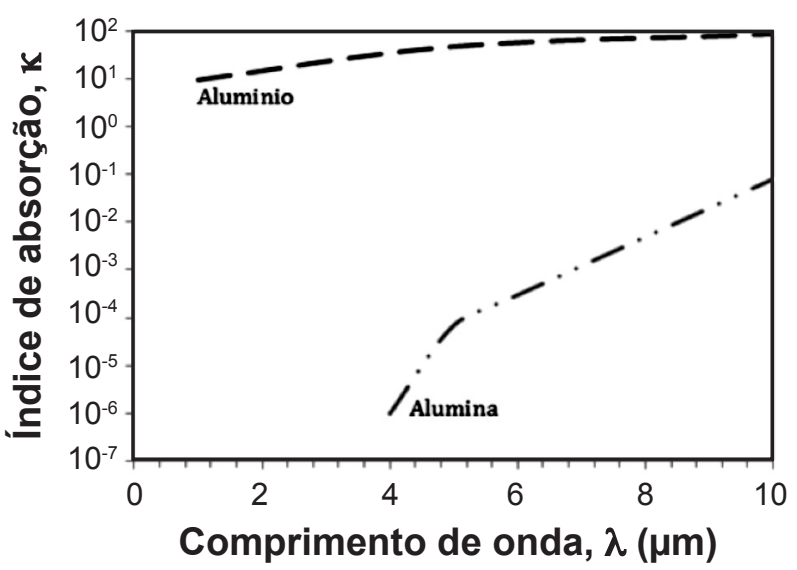

Figura 1: Índices de absorção ( $\kappa)$ do alumínio (Al) [16] e da alumina $\left(\mathrm{Al}_{2} \mathrm{O}_{3}\right)$ [17] em função do comprimento de onda $(\lambda)$. [Figure 1: Absorption index ( $\mathrm{\kappa}$ ) of aluminum (Al) [16] and alumina $\left(\mathrm{Al}_{2} \mathrm{O}_{3}\right)$ [17] as a function of the wavelength ( $\lambda$ ).]

Cada material possui uma capacidade distinta para absorver a radiação eletromagnética que incide na sua superfície, sendo definida pelo índice de absorção (k), o qual varia com o comprimento de onda considerado (Fig. 1). As tendências apresentadas na Fig. 1 estão relacionadas com o modo que estes materiais interagem com as ondas que sobre eles incide. No caso do alumínio a absorção da radiação incidente ocorre por meio da transição eletrônica dos elétrons da banda de valência para a banda de condução.

Os materiais cerâmicos apresentam uma grande diferença de energia entre a banda de valência e a de condução, como se observa na $\alpha-\mathrm{Al}_{2} \mathrm{O}_{3}$, que apresenta um intervalo de 9,4 $\mathrm{eV}$ entre as bandas [13]. Portanto, a transição eletrônica só ocorre para frequências de onda muito altas, pois estas são mais energéticas. Para a alumina tal efeito ocorre no ultravioleta que apresenta uma faixa de comprimentos de onda de $0,4 \mu \mathrm{m}$ a $0,01 \mu \mathrm{m}$ [14], ou seja, $7,5.10^{16} \mathrm{~Hz}$ a $3,0.10^{16}$, respectivamente, o que corresponde à faixa de energia de aproximadamente $3 \mathrm{eV}$ a $124 \mathrm{eV}$ [15]. Desta forma, a radiação infravermelha é incapaz de promover os elétrons presentes nos materiais cerâmicos em decorrência de sua energia associada que varia de aproximadamente $0,012 \mathrm{eV}$ a 1,6 eV. Logo, nestes compostos a absorção de radiação eletromagnética se dá por polarização (p), a qual está intimamente ligada à constante dielétrica complexa $(\epsilon)$ do material e a sua polarizabilidade $(\alpha)$, como pode ser observado nas equações D e E5 [18].

$$
\begin{gathered}
\mathrm{P}=(\epsilon-1) \cdot \varepsilon_{0} \cdot \mathrm{E} \\
\alpha=\frac{\mathrm{P}}{\mathrm{N} \cdot \mathrm{E}_{\mathrm{loc}}}
\end{gathered}
$$

sendo $\varepsilon_{0}$ a permissividade elétrica do vácuo $\left(8,854.10^{-12} \mathrm{C}^{2} / \mathrm{J} . \mathrm{m}\right)$, o campo elétrico externo $(\mathrm{N} / \mathrm{C}), \mathrm{E}_{\mathrm{loc}} \mathrm{o}$ campo elétrico local $(\mathrm{N} / \mathrm{C})$ e $\mathrm{N}$ o número de espécies polarizáveis por unidade de volume. Adicionalmente, o campo elétrico local $\left(\mathrm{E}_{\mathrm{loc}}\right)$ se relaciona com o campo elétrico externo (E) segundo a equação $\mathrm{F}$. 


$$
\mathrm{E}_{\mathrm{loc}}=\frac{\mathrm{E}}{3}(\epsilon+2)
$$

Deste modo, observa-se que quanto maior a constante dielétrica $(\epsilon)$ do material, maior a polarizabilidade $(\alpha)$ do mesmo, o que se torna perceptível ao substituir as equações $\mathrm{D}$ e $\mathrm{F}$ em $\mathrm{E}$. Cabe ainda destacar que a constante dielétrica complexa $(\epsilon)$, que é adimensional, é constituída por uma parte real $(\epsilon)$ e outra imaginária $\left(\epsilon_{1}\right)$, assim como apresentado na equação $\mathrm{G}$.

$$
\epsilon=\epsilon_{1}+\mathrm{i} \epsilon_{2}
$$

O índice de refração complexo (n') (equação H), que é composto por uma parte real denominada índice de refração (n) e uma imaginária chamada de índice de absorção (k), ambos adimensionais, está intimamente relacionado com a parte real $\left(\epsilon_{1}\right)$ e imaginária $\left(\epsilon_{2}\right)$ da constante dielétrica complexa $(\epsilon)$, pois e são funções destes dois parâmetros como segue nas equações I e J.

$$
\begin{aligned}
& \mathrm{n}^{\prime}=\mathrm{n}+\mathrm{ik} \\
& \mathrm{n}=\sqrt{\frac{\sqrt{\epsilon_{1}^{2}+\epsilon_{2}^{2}}+\epsilon_{1}}{2}} \\
& \mathrm{k}=\sqrt{\frac{\sqrt{\epsilon_{1}^{2}+\epsilon_{2}^{2}}-\epsilon_{1}}{2}}
\end{aligned}
$$

Portanto, pelas equações de $\mathrm{D}$ a $\mathrm{J}$ nota-se que a polarizabilidade $(\alpha)$ e os índices de refração (n) e absorção (k) estão relacionados diretamente por meio da constante dielétrica complexa $(\epsilon)$. No entanto, a equação explicita para esta relação não é trivial.

A polarizabilidade total é dada pela soma da contribuição da polarizabilidade eletrônica $\left(\alpha_{\varepsilon}\right)$, da polarizabilidade iônica $\left(\alpha_{\text {ion }}\right)$ e da polarizabilidade dipolar $\left(\alpha_{\text {dip }}\right)$, como descrito na equação K [18].

$$
\left(\mathrm{N}_{\mathrm{e}} \cdot \alpha_{\mathrm{e}}+\mathrm{N}_{\text {ion }} \cdot \alpha_{\text {ion }}+\mathrm{N}_{\text {dip }} \cdot \alpha_{\text {dip }}\right) 3 \cdot \varepsilon_{0} \cdot \frac{\epsilon-1}{\epsilon+2}
$$

Na Fig. 2 é possível observar a ação de cada uma dessas polarizações nas componentes real $\left(\epsilon_{1}\right)$ e imaginária $\left(\epsilon_{2}\right)$ da constante dielétrica $(\epsilon)$, nas faixas de frequências em que ocorrem.

\subsubsection{Polarização eletrônica $\left(\alpha_{\varepsilon}\right)$}

Essa polarização é proveniente do deslocamento da nuvem eletrônica em relação ao núcleo do átomo, sendo que este fenômeno ocorre para todas as frequências de onda, mas apresenta uma redução significativa para aquelas superiores $\mathrm{a} 10^{15} \mathrm{~Hz}$ (ultravioleta) [14, 18]. Portanto, este tipo de polarização é de interesse para frequências de onda correspondentes ao infravermelho e visível, que estão compreendidas na faixa entre $10^{11} \mathrm{~Hz}$ e $7,9.10^{14} \mathrm{~Hz}[12,14]$, e são emitidas a altas temperaturas, como será explicado

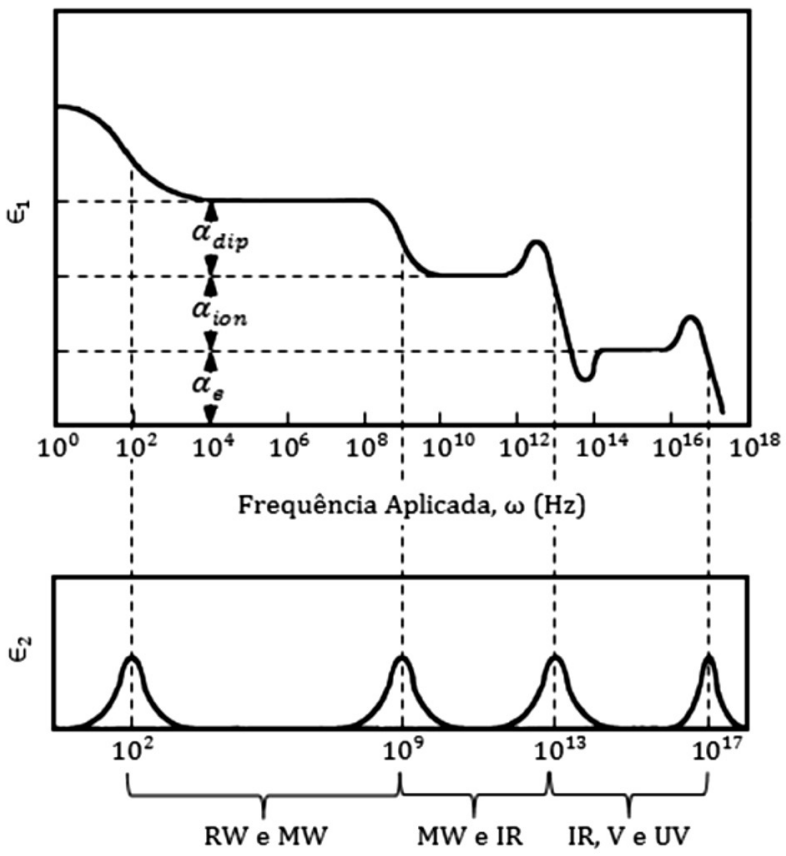

Figura 2: Variação da parte real $\left(\epsilon_{1}\right)$ e imaginária $\left(\epsilon_{2}\right)$ da constante dielétrica $(\epsilon)$ em função da frequência de onda aplicada $(\mathrm{Hz})$ destacando os tipos de polarização que ocorrem em cada faixa de frequência e a classe de onda a que correspondem [18] [RW (ondas de rádio), MW (micro-ondas), IR (infravermelho), V (visível) e UV (ultravioleta)].

[Figure 2: Variation of the real part $\left(\epsilon_{1}\right)$ and the imaginary one $\left(\epsilon_{2}\right)$ of the dielectric constant $(\in)$ as a function of the applied wave frequency $(\mathrm{Hz})$, highlighting the types of polarization occurring for each frequency range and the corresponding wave class [18] [RW (radio waves), $M W$ (microwave), IR (infrared), V(visible) and UV(ultraviolet)].]

no decorrer deste artigo. Quando se torna possível assumir que o campo aplicado (E) é igual ao local, condição válida no caso de gases diluídos, a parte real $\left(\epsilon_{1}\right)$ e a imaginária $\left(\epsilon_{2}\right)$ da constante dielétrica assumem os valores dados pelas equações 12 e 13 respectivamente.

$$
\begin{aligned}
& \epsilon_{1}(\omega)=1+\frac{Z_{\mathrm{i}} \cdot \mathrm{e}^{2} \cdot \mathrm{N} \cdot\left(\omega_{0}^{2}-\omega^{2}\right)}{\varepsilon_{0} \cdot \mathrm{m}_{\mathrm{e}}\left[\left(\omega_{0}^{2}-\omega^{2}\right)^{2}+\mathrm{f}^{2} \cdot \omega^{2}\right]} \\
& \epsilon_{2}(\omega)=\frac{Z_{\mathrm{i}} \cdot \mathrm{e}^{2} \cdot \mathrm{N} \cdot \omega \cdot \mathrm{f}}{\varepsilon_{0} \cdot \mathrm{m}_{\mathrm{e}}\left[\left(\omega_{0}^{2}-\omega^{2}\right)^{2}+\mathrm{f}^{2} \cdot \omega^{2}\right]}
\end{aligned}
$$

sendo $\mathrm{Z}_{\mathrm{i}} \mathrm{o}$ número atômico, a carga do elétron $(\mathrm{C}), \mathrm{f}$ o coeficiente de atrito $(\mathrm{rad} / \mathrm{s}), \omega_{0}$ a frequência natural de vibração do sistema $(\mathrm{Hz})$, também chamada de frequência de ressonância, $\omega$ freqüência do campo aplicado $(\mathrm{Hz})$ e $\mathrm{m}_{\mathrm{e}} \mathrm{a}$ massa do elétron $(\mathrm{kg})$. Desta forma, quanto mais a frequência da onda incidente tende para a frequência natural de vibração $\left(\omega_{0}\right)$, maior será a absorção observada, pois o sistema torna-se ressonante. Logo, a constante dielétrica alcança seu máximo (Figs. 3 e 4), e consequentemente o mesmo ocorre com a polarizabilidade (equações E, L e M), com o índice refração (n) e o de absorção (k) (Fig. 5). Além disso, considerando- 


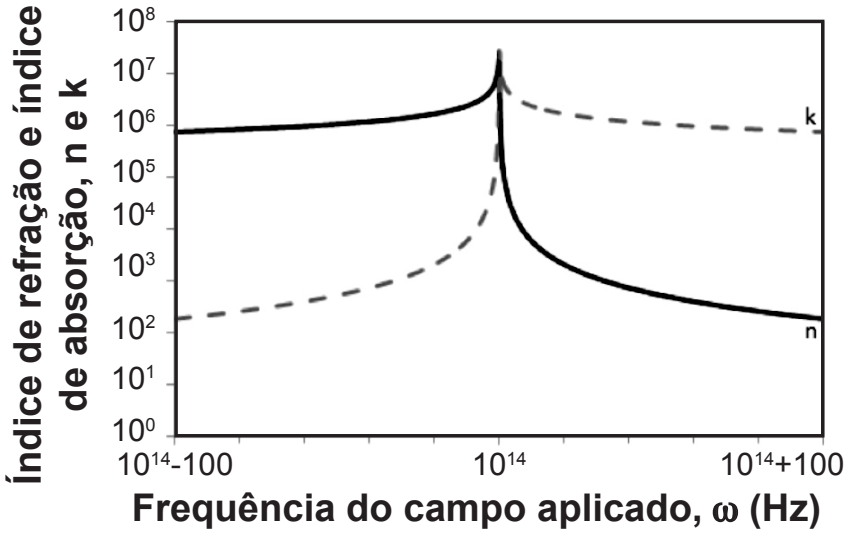

Frequência do campo aplicado, $\omega(\mathrm{Hz})$

Figura 3: Comportamento da parte real da constante dielétrica $\left(\epsilon_{1}\right)$ para a polarização eletrônica $(\alpha)$ calculada pela equação $\mathrm{L}$, adotando-se $\mathrm{f}=0,1 \mathrm{rad} / \mathrm{s}, \mathrm{Z}_{\mathrm{i}}=6, \mathrm{~N}=1 \mathrm{~mol} / \mathrm{m}^{3}=6,02.10^{23}$ espécies $/ \mathrm{m}^{3} \mathrm{e} \omega_{0}=10.10^{14} \mathrm{~Hz}$ em função da frequência do campo aplicado $(\omega)$.

[Figure 3: Behavior of the real part of dielectric constant $\left(\epsilon_{1}\right)$ for the electronic polarization $\left(\alpha_{e}\right)$ calculated by equation $L$, assuming $f=0.1 \mathrm{rad} / \mathrm{s}, \mathrm{Z}_{\mathrm{i}}=6, N=1 \mathrm{~mol} / \mathrm{m}^{3}=6.02 .10^{23} \mathrm{species} / \mathrm{m}^{3}$ and $\omega_{0}=$ $10.10^{14} \mathrm{~Hz}$ as a function of the applied field frequency $\left.(\omega).\right]$

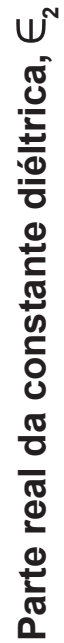

$1,2.10^{15}$

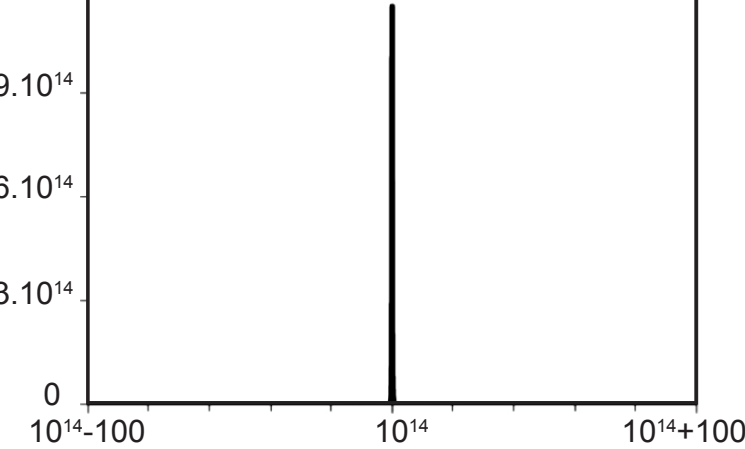

Frequência do campo aplicado, $\omega(\mathrm{Hz})$

Figura 4: Comportamento da parte imaginária da constante dielétrica $\left(\epsilon_{2}\right)$ para a polarização eletrônica $\left(\alpha_{e}\right)$ calculada pela equação $M$, adotando-se $\mathrm{f}=0,1 \mathrm{rad} / \mathrm{s}, \mathrm{Z}_{\mathrm{i}}=6, \mathrm{~N}=1 \mathrm{~mol} / \mathrm{m}^{3}=6,02.10^{23}$ espécies $/ \mathrm{m}^{3}$ e $\omega_{0}=10,10^{14} \mathrm{~Hz}$ em função da frequência do campo aplicado $(\omega)$. [Figure 4: Behavior of the imaginary part of dielectric constant $\left(\epsilon_{2}\right)$ for the electronic polarization $\left(\alpha_{e}\right)$ calculated by equation $M$, adopting $f=0.1 \mathrm{rad} / \mathrm{s}, \mathrm{Z}_{\mathrm{i}}=6, N=1 \mathrm{~mol} / \mathrm{m}^{3}=6.02 .10^{23} \mathrm{species} / \mathrm{m}^{3}$ and $\omega_{0}=10.10^{14} \mathrm{~Hz}$ as a function of the applied field frequency $\left.(\omega).\right]$

se que o campo elétrico local $\left(\mathrm{E}_{\text {loc }}\right)$ é igual ao externo $(\mathrm{E})$, então para as situações em que $\omega_{0}$ é muito maior que $\omega$, a polarizabilidade eletrônica $\left(\alpha_{\mathrm{e}}\right)$ é calculada pela equação $\mathrm{N}$ [18]. Esta expressão matemática é decorrente da eliminação de $\omega_{0}$ e dos termos que acompanham $\omega$ da equação L, combinando-se este resultado com as equações D e E.
Figura 5: Comportamento do índice de refração (n) e absorção (k) para a polarização eletrônica $\left(\alpha_{\mathrm{e}}\right)$ calculados respectivamente pelas equações I e J, adotando-se $\mathrm{f}=0,1 \mathrm{rad} / \mathrm{s}, \mathrm{Z}_{\mathrm{i}}=6, \mathrm{~N}=1 \mathrm{~mol} / \mathrm{m}^{3}=$ $6,02.10^{23}$ espécies $/ \mathrm{m}^{3} \mathrm{e} \omega_{0}=10.10^{14} \mathrm{~Hz}$ para o cálculo da parte real $\left(\epsilon_{1}\right)$ e imaginária $\left(\epsilon_{2}\right)$ da constante dielétrica $(\epsilon)$ e em função da frequência do campo aplicado $(\omega)$.

[Figure 5: Behavior of the refractive index (n) and absorption index $(k)$ for the electronic polarization $\left(\alpha_{\mathrm{e}}\right)$ calculated by equations $I$ and $J$, respectively, assuming $f=0.1 \mathrm{rad} / \mathrm{s}, Z_{i}=6, N=1 \mathrm{~mol} / \mathrm{m}^{3}$ $=6.02 .10^{23}$ species $/ \mathrm{m}^{3}$ and $\omega_{0}=10.10^{14} \mathrm{~Hz}$ for calculating the real $\left(\epsilon_{1}\right)$ and imaginary $\left(\epsilon_{2}\right)$ parts of dielectric constant as a function of the applied field frequency $(\omega)$.]

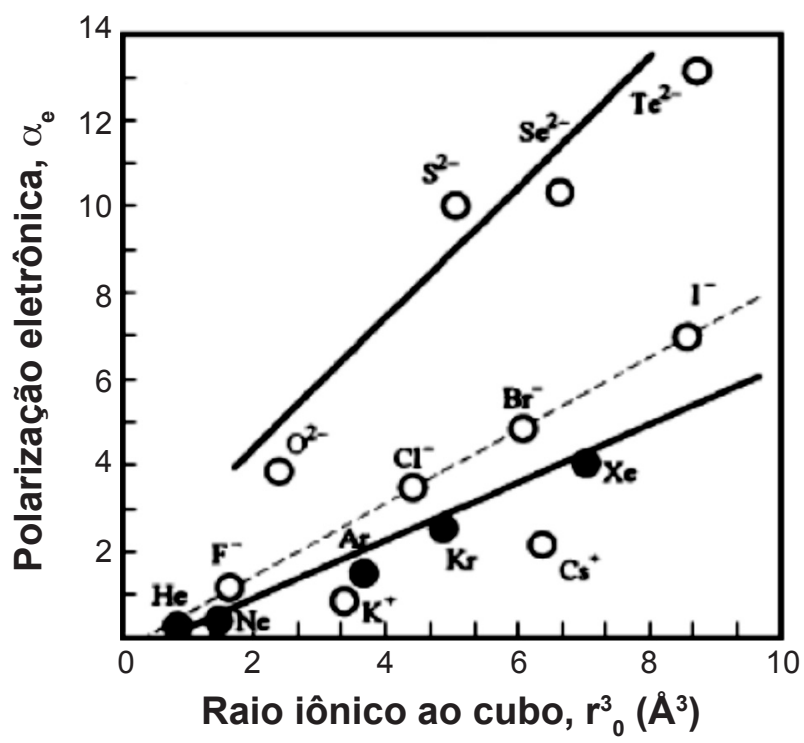

Figura 6: Polarizabilidade eletrônica $\left(\alpha_{e}\right)$ de íons em função do seu raio elevado a terceira potência $\left(\mathrm{r}_{0}^{3}\right)$ [18].

[Figure 6: Electronic polarizability $\left(\alpha_{\mathrm{e}}\right)$ of selected ions as a function of their radii to the third power $\left(r^{3}\right)$ [18].]

$$
\alpha_{\mathrm{e}}=\frac{\mathrm{Z}_{\mathrm{i}} \mathrm{e}^{2}}{\mathrm{~m}_{\mathrm{e}} \omega_{0}^{2}}
$$

No entanto, neste caso esta equação pode ser aproximada pela expressão O [18].

$$
\alpha_{\mathrm{e}} \approx 4 \cdot \pi \cdot \varepsilon_{0} \mathrm{r}_{0}^{3}
$$

na qual $\mathrm{r}_{0}$ é o raio do átomo ou íon. Portanto, a equação 
$\mathrm{O}$ indica que quanto maior o átomo ou íon, maior sua polarizabilidade eletrônica $\left(\alpha_{\mathrm{e}}\right)$. Este fenômeno pode ser visto na Fig. 6. Como o tamanho do íon está diretamente ligado a sua carga, tal fator deve ser considerado, pois quanto mais negativa a carga do íon, maior o seu raio iônico, o que resulta numa maior polarizabilidade. Por consequência, quanto mais positiva a carga do íon menos polarizável ele se torna.

A blindagem da carga nuclear também influencia na polarizabilidade da espécie considerada, pois ao aumentar a carga nuclear efetiva, mais presa encontra-se a eletrosfera ao redor do núcleo. Consequentemente, elementos que contem subníveis d ou $\mathrm{f}$ tendem a ser menos polarizáveis [18].

\subsubsection{Polarização iônica $\left(\alpha_{\text {เon }}\right)$}

Este tipo de polarização ocorre quando os íons positivos e negativos se deslocam de sua posição de equilíbrio, adquirindo uma nova configuração na qual o centro de cargas positivas não coincide com o centro de cargas negativas. No que se refere à frequência de ressonância iônica $\left(\omega_{\text {ton }}\right)$, a qual está associada ao máximo de absorção, esta se encontra na faixa do infravermelho [18] de $10^{11} \mathrm{~Hz}$ a $3,9.10^{14} \mathrm{~Hz}$. Para este tipo de polarização a parte real $\left(\epsilon_{1}\right)$ e imaginária $\left(\epsilon_{2}\right)$ da constante dielétrica $(\in)$ de um composto são respectivamente expressas pelas equações $\mathrm{P}$ e Q.

$$
\begin{aligned}
& \epsilon_{1}(\omega)=1+\frac{\left(Z_{\mathrm{i}} \cdot e\right)^{2} \cdot \mathrm{N}_{\mathrm{ion}} \cdot\left(\omega_{\mathrm{ion}}^{2}-\omega^{2}\right)}{\varepsilon_{0} \cdot \mathrm{M}_{\mathrm{r}} \cdot\left[\left(\omega_{\text {ion }}^{2}-\omega^{2}\right)^{2}+\underset{\text { ion }}{\mathrm{f}^{2}} \cdot \omega^{2}\right]} \\
& \epsilon_{2}(\omega)=\frac{\left(Z_{\mathrm{i}} \cdot \mathrm{e}\right)^{2} \cdot \mathrm{N}_{\mathrm{ion}} \cdot \omega \cdot \mathrm{f}_{\mathrm{ion}}^{2}}{\varepsilon_{0} \cdot \mathrm{M}_{\mathrm{r}} \cdot\left[\left(\omega_{\text {ion }}^{2}-\omega^{2}\right)^{2}+\underset{\text { ion }}{\mathrm{f}} \cdot \omega^{2}\right]}
\end{aligned}
$$

sendo o coeficiente de atrito do sistema iônico ( $\mathrm{rad} / \mathrm{s})$ e a massa reduzida do sistemadada pela expressão $\mathrm{R}$.

$$
\mathrm{M}_{\mathrm{r}}=\frac{\mathrm{m}_{\mathrm{c}} \cdot \mathrm{m}_{\mathrm{a}}}{\mathrm{m}_{\mathrm{c}}+\mathrm{m}_{\mathrm{a}}}
$$

na qual $\mathrm{m}_{\mathrm{c}} \mathrm{e} \mathrm{m}_{\mathrm{a}}$ são massa do cátion $\mathrm{e}$ do ânion, respectivamente. Portanto, pelas equações $\mathrm{P}$ e Q observase que as componentes real $\left(\epsilon_{1}\right)$ e imaginária $\left(\epsilon_{2}\right)$ da constante dielétrica $(\in)$ são inversamente proporcionais à massa reduzida do sistema $\left(\mathrm{M}_{\mathrm{r}}\right)$ e a frequência natural de vibração $\left(\omega_{\text {ion }}\right)$ do mesmo, sendo esta última relacionada às forças de Coulomb existentes. Consequentemente, como a polarizabilidade iônica $\left(\alpha_{\text {ion }}\right)$ diminui com a redução de $\epsilon_{1}$ $\mathrm{e} \epsilon_{2}$, ligações mais fortes reduzem a polarizabilidade iônica $\left(\alpha_{\text {ion }}\right)$, pois geram $\omega_{\text {ion }}$ mais elevados.

\subsubsection{Polarização dipolar $\left(\alpha_{\text {dip }}\right)$}

Este tipo de polarização ocorre apenas em baixas frequências ${ }^{17}$, geralmente abaixo de $10^{10} \mathrm{~Hz}$, como para micro-ondas ou ondas de rádio, logo sua interação com a radiação térmica não é significativa, pois esta possui frequências superiores a $10^{12} \mathrm{~Hz}$.

\subsection{Refletividade}

A equação de Fresnel descreve o comportamento de uma onda plana e homogênea, indicando a parcela desta que é refletida em função do ângulo de incidência $\left(\theta_{1}\right)$ ao passar de um meio não absorvedor e com índice de refração $\mathrm{n}_{1}$, para outro, que pode ou não ser um absorvedor e que apresenta um índice de refração $\mathrm{n}_{2} \mathrm{e}$ índice de absorção $\mathrm{k}_{2}$ [12]. Além disso, a equação considera que a superfície de contanto entre os meios é uma superfície especular, ou seja, isenta de defeitos e rugosidade, o que leva a um ângulo de reflexão igual ao de incidência.

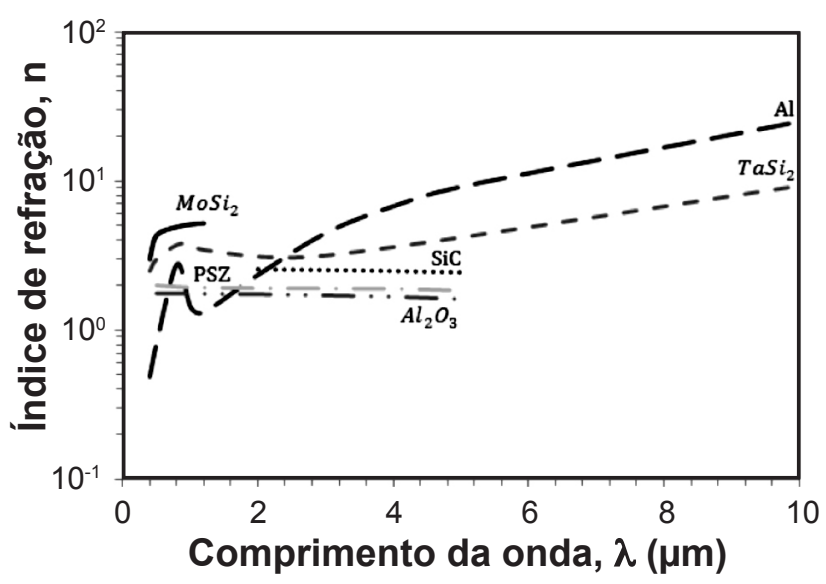

Figura 7: Índice de refração (n) $[16,17,19,20]$ em função do comprimento de onda $(\lambda)$ para os materiais abordados ao longo do artigo.

[Figure 7: Refractive index (n) [16, 17, 19, 20] as a function of the wavelength ( $\lambda$ ) for the materials presented in the paper.]

Segundo Fresnel, a refletividade da parte polarizada paralela $\left(\rho_{\|}\right)$ao plano de incidência e da parte polarizada perpendicular $\left(\rho_{\mathrm{L}}\right)$ a este, podem ser descritas pelas equações $\mathrm{S}$ e $\mathrm{T}$.

$$
\begin{aligned}
& \rho_{\|}=\frac{\left(p-n_{1} \cdot \sin \left(\theta_{1}\right) \cdot \tan \left(\theta_{1}\right)\right)^{2}+q^{2}}{\left(p+n_{1} \cdot \sin \left(\theta_{1}\right) \cdot \tan \left(\theta_{1}\right)\right)^{2}+q^{2}} \cdot \rho_{L} \\
& \rho_{L}=\frac{\left(n_{1} \cdot \cos \left(\theta_{1}\right)-p\right)^{2}+q^{2}}{\left(n_{1} \cdot \cos \left(\theta_{1}\right)+p\right)^{2}+q^{2}}
\end{aligned}
$$

sendo que os parâmetros p e q podem ser expressos pelas equações $\mathrm{U}$ e $\mathrm{V}$.

$$
\mathrm{p}^{2}=\frac{1}{2} \cdot\left[\sqrt{\left(\mathrm{n}_{2}^{2}-\mathrm{k}_{2}^{2}-\mathrm{n}_{1}^{2}\left(\sin \theta_{1}\right)^{2}\right)^{2}+4 \cdot \mathrm{n}_{2}^{2} \cdot \mathrm{k}_{2}^{2}+\left(\mathrm{n}_{2}^{2}-\mathrm{k}_{2}^{2}-\mathrm{n}_{1}^{2} \cdot\left(\sin \left(\theta_{1}\right)\right)^{2}\right)}\right]
$$

$\mathrm{q}^{2}=\frac{1}{2} \cdot\left[\sqrt{\left(\mathrm{n}_{2}^{2}-\mathrm{k}_{2}^{2}-\mathrm{n}_{1}^{2}\left(\sin \theta_{1}\right)^{2}\right)^{2}+4 \cdot \mathrm{n}_{2}^{2} \cdot \mathrm{k}_{2}^{2}-\left(\mathrm{n}_{2}^{2}-\mathrm{k}_{2}^{2}-\mathrm{n}_{1}^{2} \cdot\left(\sin \left(\theta_{1}\right)\right)^{2}\right)}\right](\mathrm{V})$ 
A refletividade para a onda não polarizada é a média aritmética da reflexão da parte polarizada paralelamente $\left(\rho_{\| I}\right)$ a interface de incidência e da reflexão da parte polarizada perpendicularmente $\left(\rho_{\mathrm{L}}\right)$ a mesma, como pode ser observado pela equação $X$.

$$
\mathrm{R}=\frac{\left(\rho_{\| 1}+\rho_{\mathrm{L}}\right)}{2}
$$

Portanto, para efetuar tais cálculos basta ter o índice de refração (n) e o índice de absorção (k) do material para o comprimento de onda $(\lambda)$ desejado, sendo que a Fig. 7 apresenta os índices de refração ( para os compostos que serão discutidos ao longo deste artigo. Portanto, por meio das equações $\mathrm{S}$ a X [12], torna-se possível simular a refletividade de diversos compostos em função do ângulo de incidência $\left(\theta_{1}\right)$ da onda, como exemplifica a Fig. 8, na qual para os sistemas ar-alumínio e ar-alumina se observa a porcentagem refletida de uma radiação de $5 \mu \mathrm{m}$ tanto a não polarizada $(\mathrm{R})$ como para suas componentes polarizadas $\left(\rho_{\| 1}\right)$ e $\left(\rho_{\mathrm{L}}\right)$.

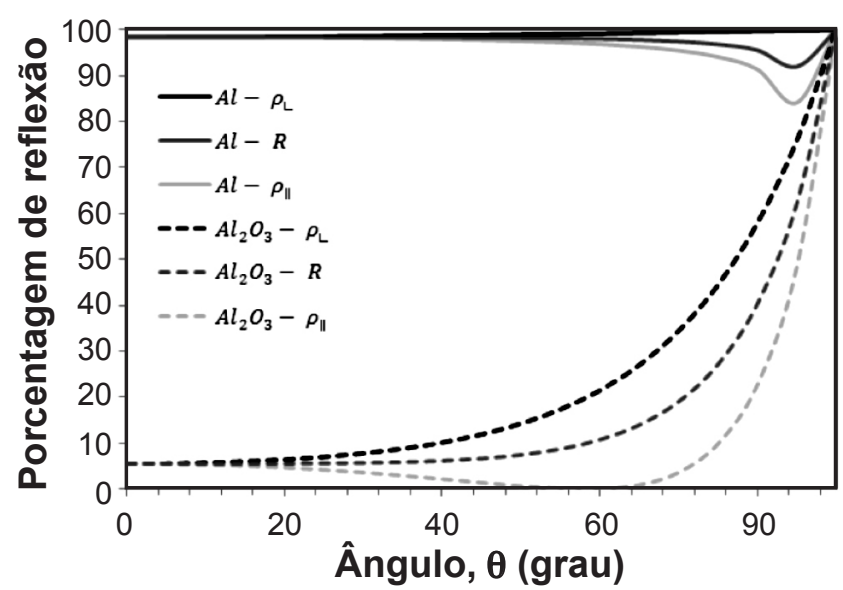

Figura 8: Porcentagem de reflexão para uma radiação de $5 \mu$ m não polarizada $(\mathrm{R})$ e suas componentes polarizadas perpendicularmente $\left(\rho_{\mathrm{L}}\right)$ e paralelamente $\left(\rho_{\|}\right)$ao plano de incidência, para o sistema aralumínio $(\mathrm{Al})$ e o ar-alumina $\left(\mathrm{Al}_{2} \mathrm{O}_{3}\right)$, calculados pelas equações de $\mathrm{S}$ a X com base nos seus respectivos índices de refração (n) $[16,17$, $19,20]$ e absorção $(\mathrm{k})[16,17]$.

[Figure 8: Percentage of reflection (R) for anon-polarized $5 \mu \mathrm{m}$ radiationand its perpendicular $\left(\rho_{\mathrm{L}}\right)$ and parallel $\left(\rho_{\|}\right)$polarized components to the plane of incidence for the air-aluminum system (Al) and the air-alumina one $\left(\mathrm{Al}_{2} \mathrm{O}_{3}\right)$, calculated by equations $\mathrm{S}-\mathrm{X}$ with their respective refractive (n) $[16,17,19,20]$ and absorption indexes (k) [16, 17].]

\subsection{Emissividade}

A lei de Stefan-Boltzmann define que a energia total irradiada por unidade de área superficial $(\tau)\left[\mathrm{W} / \mathrm{m}^{2}\right]$ de um corpo negro, ou seja, de um perfeito emissor, é diretamente proporcional à temperatura (), como expresso na equação $\mathrm{Y}$ [12].

$$
\tau=\sigma \cdot T^{4}
$$

na qual $\sigma$ é a constante de Stefan-Boltzmann $\left(1,3806503.10^{-23} \mathrm{~J} / \mathrm{K}\right)$ e T é a temperatura expressa em Kelvin. Tal equação pode ser generalizada para o caso não ideal [no qual o material é um corpo cinza, ou seja, possui uma emissividade $(\varepsilon)$ menor que um por meio da incorporação desta propriedade na equação Y como descrito na equação $Z$.

$$
\tau=\varepsilon . \sigma \cdot T^{4}
$$

A energia total $(\tau)$ irradiada corresponde à soma da emissão em todos os comprimentos de onda $(\lambda)$, sendo que o valor da emissão varia de um comprimento de onda $(\lambda)$ para outro e em função da temperatura $(\mathrm{T})$ do corpo, como expresso pela lei de Planck [12]. Diversas são as formas de se expressar a equação que descreve a lei de Planck, sendo que para um corpo negro, com superfície limitada por um meio transparente, por exemplo, o vácuo, tais expressões podem ser apresentadas em função da frequência $(v)$ e do comprimento de onda $(\lambda)$, como segue apresentado abaixo.

$$
\begin{aligned}
& \mathrm{W}_{\lambda}(\mathrm{T}) \frac{2 \cdot \pi \cdot h \cdot v^{3} \cdot \mathrm{n}^{2}}{\mathrm{c}^{2}} \cdot \frac{1}{\mathrm{e}^{\frac{h \cdot v}{\sigma \cdot \mathrm{T}}}-1} \\
& \mathrm{~W}_{\lambda}(\mathrm{T})=\frac{2 \cdot \pi \cdot h \cdot \mathrm{c}^{2}}{\mathrm{n}^{2} \cdot \lambda^{5}} \cdot \frac{1}{\mathrm{e}^{\frac{\mathrm{h} \cdot \mathrm{c} \cdot \mathrm{c} \cdot \mathrm{T}}{\mathrm{n} \cdot \mathrm{T}}}-1}
\end{aligned}
$$

sendo h a constante de Planck $\left(6,6260693.10^{-34} \mathrm{~J} . \mathrm{s}\right)$ e a velocidade da luz no vácuo $(\mathrm{m} / \mathrm{s})$. Por meio da equação $A B$ e considerando-se o material no vácuo $(\mathrm{n}=1)$, é possível obter-se a Fig. 9 que é denominada espectro de Planck, a qual contabiliza a potência emissiva (W) $\left[(\mathrm{W} . \mu \mathrm{m}) / \mathrm{m}^{2}\right]$ do corpo em função da sua temperatura $(\mathrm{T})$ e do comprimento de onda $(\lambda)$.

Observando-se a Fig. 9, fica evidente que um aumento da temperatura leva a uma maior quantidade de energia emitida e a um deslocamento do pico de emissão para

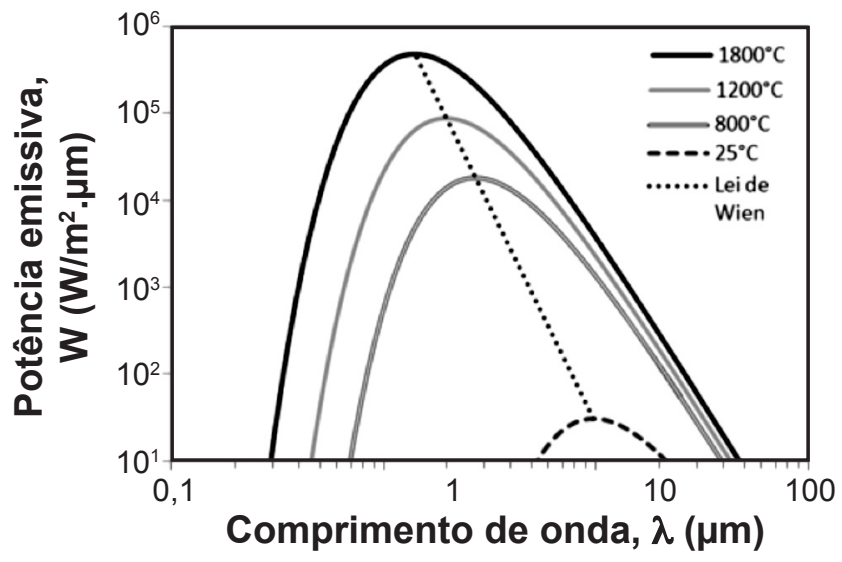

Figura 9: Espectro de Planck da potência emissiva (W) em função da temperatura e do comprimento de onda $(\lambda)[21]$.

[Figure 9: Planck's spectrum of emissive power $(W)$ as a function of temperature and wavelength ( $\lambda$ ) [21].] 
comprimentos de onda mais baixos. Este comportamento pode ser descrito matematicamente pela lei de Wien que calcula o comprimento de onda $(\lambda)$ do pico de emissão numa dada temperatura $(\mathrm{T})$, segundo a equação $\mathrm{AC}$.

$$
\lambda=\frac{2897,77}{\mathrm{~T}}(\mu \mathrm{m})
$$

\subsubsection{Lei de Kirchhoff}

Para melhor compreender a emissividade $(\varepsilon)$ e sua relação com as demais propriedades ópticas é necessário o auxílio da lei de Kirchhoff [12], a qual postula que no equilíbrio térmico a emissividade total apresentada pelo material é igual a sua absorção total. Desta forma concluise que, para um corpo opaco a equação $\mathrm{AD}$ é válida, pois a transmissão é zero. Vale lembrar que por definição um corpo opaco é aquele que absorve toda a radiação incidente, ou seja, transmissão nula.

$$
\varepsilon=\mathrm{A}=1-\mathrm{R}
$$

Portanto, nota-se que a absorção (A) e a refletividade (R) são fenômenos concorrentes e, além disso, que a quantia de energia absorvida (A) pelo material é igual à da energia emitida $(\varepsilon)$ pelo mesmo. Consequentemente, mesmo fora das condições de contorno da lei de Kirchhoff, ou seja, fora do equilíbrio, a absorção (A) e a emissividade (ع) também devem estar associadas, apesar de sua relação não ser expressa pela equação $\mathrm{AD}$.

2. Tintas de alta emissividade aplicadas aos veículos espaciais

Na década de 70 a NASA,visando possibilitar uma reentrada segura para suas espaçonaves, desenvolveu uma primeira geração de tintas de alta emissividade com o objetivo de serem aplicadas sobre o substrato cerâmico externo do sistema de proteção térmico dos ônibus espaciais. A idéia central dessa tinta é que ao ser aquecida devido ao atrito aerodinâmico, ela passe a emitir radiação térmica, dissipando calor para o ambiente. Dessa forma, a primeira geração foi constituída por uma matriz de vidro borossilicato, tendo como agente emissor principal o carbeto de silício (SiC) $[1,2]$. No entanto, na década de 90, a NASA optou por trocar o agente emissor, passando a ser o dissiliceto de molibdênio $\left(\mathrm{MoSi}_{2}\right)$. Tal composto corresponde a uma classe de materiais denominada intermetálicos, a qual nas últimas décadas vem despertando grande interesse dado suas novas aplicações [22]. Além disso, o $\mathrm{MoSi}_{2}$ é refratário com uma temperatura de fusão de $2030^{\circ} \mathrm{C}[23,24]$.

Em 2010 a quantidade utilizada de dissiliceto de molibdenio na formulação foi reduzida dando lugar ao dissiliceto de tântalo $\left(\mathrm{TaSi}_{2}\right)$ [25], constituindo assim uma terceira geração cujas proporções de compostos utilizados estão descritas na Fig. 10. Além disso, a matriz de vidro de borossilicato foi mantida ao longo de todas as gerações de tintas desenvolvidas pela NASA, visto que cumpre a

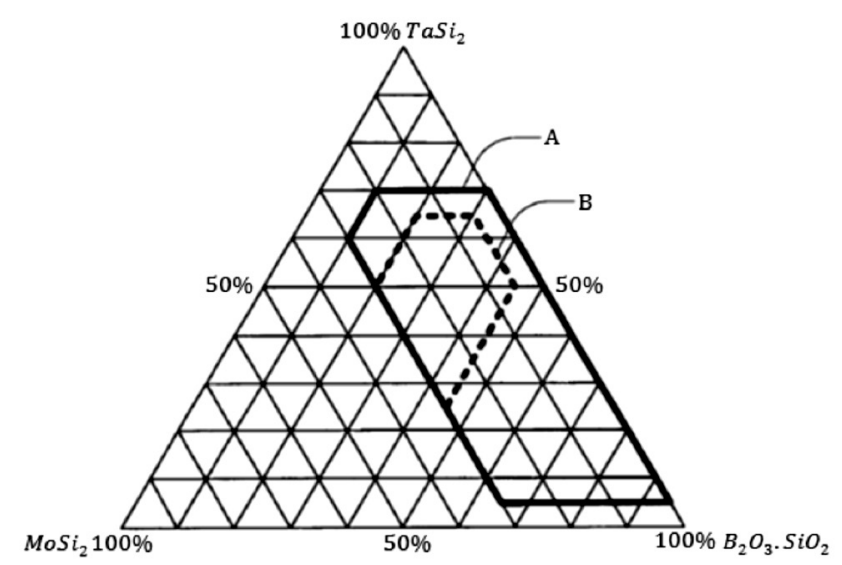

Figura 10: Diagrama de fases ternário indicativo das porcentagens utilizadas de cada um dos compostos que constituem a terceira geração de tintas de alta emissividade da NASA. A área marcada como A representa a faixa de composições com resultados satisfatórios e aquela demarcada como B consiste nos melhores resultados obtidos [3].

[Figure 10: Ternary phase diagram with the percentages of each compound used for the third generation of NASA high emissivity coating. The area highlighted with $A$ represents the group of compositions with suitable results, and the Bone, the best results attained [3].]

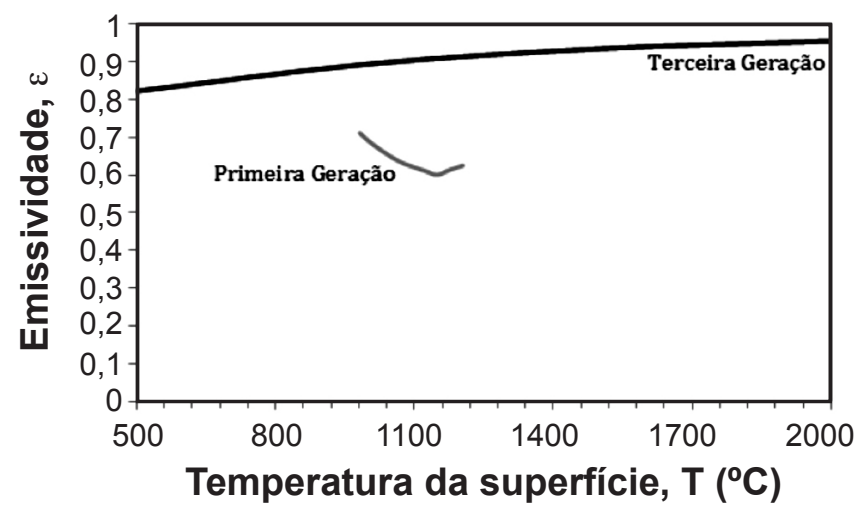

Figura 11: Emissividade da primeira ( $\mathrm{SiC}+$ borossilicato) [2] e terceira ( $\mathrm{MoSi}_{2}+\mathrm{TaSi}_{2}+$ borossilicato) [3] gerações de tintas de alta emissividade da NASA em função da temperatura (T).

[Figure 11: Emissivity of first (SiC+ borosilicate) [2] and third( $\mathrm{MoSi}_{2}+\mathrm{TaSi}_{2}+$ borosilicate) [3] generations of NASA's high emissivity coatings as a function of the temperature (T).]

função de evitar a oxidação dos dissilicetos presentes [26]. $\mathrm{O} \mathrm{MoSi}_{2}$ é também utilizado na produção de elementos de aquecimento para fornos de alta temperatura, sendo que neste caso, a formação in-situ de uma camada de sílica vítrea protege a sua oxidação [26]. As medidas de emissividade realizadas pela NASA estão apresentadas nas Figs. 11 e 12, sendo que a Fig. 11 refere-se à emissividade da primeira e terceira gerações de tintas de alta emissividade em função da temperatura, e a Fig. 12 refere-se à emissividade da terceira geração em função do comprimento de onda $(\lambda)$ da radiação eletromagnética. Observando-se a Fig. 11, fica clara a evolução da primeira para a terceira geração de tintas de alta emissividade produzidas pela NASA, pois é considerável o 


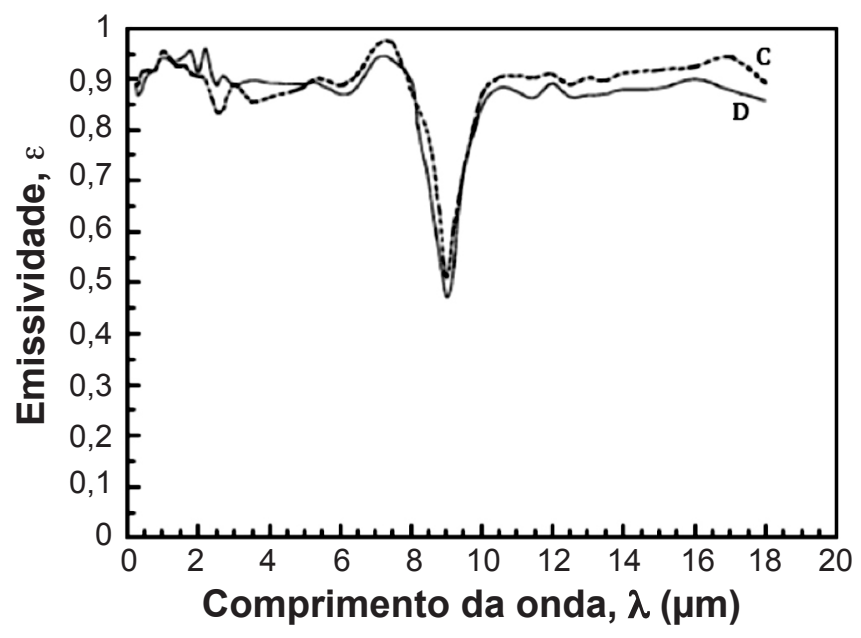

Figura 12: Emissividade da terceira geração de tintas de alta emissividade da NASA em função do comprimento de onda $(\lambda)$, onde $\mathrm{C}$ e $\mathrm{D}$ representam respectivamente a tinta antes e depois de tratada termicamente com plasma [3].

[Figure 12: Emissivity of the third generation of NASA's high emissivity coating as a function of wavelength $(\lambda)$, where $C$ and $D$ represent the coating before and after plasma heat treatment, respectively [3].]

aumento de emissividade entre as composições, o que leva a uma maior emissão total de energia como descrito pela equação $Z$. A queda de emissividade entre os comprimentos de onda de $8 \mu \mathrm{m}$ e $10 \mu \mathrm{m}$ (Fig. 12) evidencia a ausência de compostos cujo valor de $\varepsilon$ é alto nesta faixa de comprimentos de onda, sendo que, na utilização destas tintas nos veículos espaciais este aspecto não é um problema. Tais elevados comprimentos de onda influenciam pouco na emissividade total uma vez que eles estão distantes do pico de emissão nas temperaturas de trabalho desejadas, ou seja, aqueles acima de $500{ }^{\circ} \mathrm{C}$ para os quais o pico de emissão localiza-se nos comprimentos de onda inferiores a $3,75 \mu \mathrm{m}$.

\section{RESULTADOS E DISCUSSÃO}

\section{Polarização e índice de absorção}

Por meio dos conceitos de polarização abordados anteriormente torna-se possível comparar diferentes compostos cerâmicos quanto a sua polarizabilidade $(\alpha)$ e, com base nesta predizer qual deve apresentar um maior índice de absorção $(\mathrm{k})$, pois maior $\alpha$ resulta num maior valor de k (equações J e K). Sendo assim, como segue na Fig. 13, desconsiderando-se a polarizabilidade iônica $\left(\alpha_{\text {ion }}\right)$ e estimando a polarizabilidade eletrônica $\left(\alpha_{\mathrm{e}}\right)$ (equação O) [a qual é predominante nas faixas de frequência acima de $10^{12} \mathrm{~Hz}$ (Fig. 2), ou seja, para comprimentos de onda correspondentes ao infravermelho] para os íons que constituem o carbeto de silício, a alumina e a zircônia parcialmente estabilizada (PSZ), torna-se evidente qual desses materiais deve apresentar um maior índice de absorção (k). Analisando os dados da Fig. 13 nota-se que a polarizabilidade do zircônio é superior a do alumínio, logo a zircônia $\left(\mathrm{ZrO}_{2}\right)$ dopada com ítria (PSZ) deve absorver mais radiação que a alumina, o que está de acordo com os dados apresentados na Fig. 14, como pode ser observado por meio do maior índice de absorção da zircônia. Já a absorção do carbeto de silício pode ser explicada pelo elevado valor de polarizabilidade do carbono, que além de compensar o baixo valor desta constante para o silício supera em muito a dos demais íons apontados. Este fato explica o maior índice de absorção $(\mathrm{k})$ apresentado pelo carbeto de silício até o

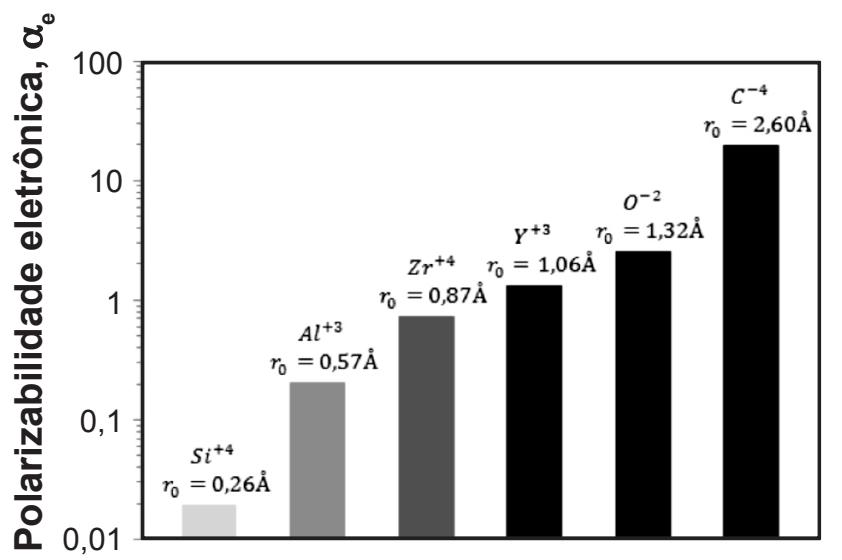

Figura 13: Estimativa da polarizabilidade eletrônica $\left(\alpha_{\mathrm{e}}\right)$ para as espécies iônicas (cátions e ânions) que formam os compostos cerâmicos carbeto de silício $\left(\mathrm{Si}^{4+} \mathrm{e} \mathrm{C}^{4-}\right)$, alumina $\left(\mathrm{Al}^{3+} \mathrm{e} \mathrm{O}^{2-}\right)$ e zircônia parcialmente estabilizada com ítria (PSZ) $\left(\mathrm{ZR}^{4+}, \mathrm{O}^{2-} \mathrm{e}^{3+}\right)$, calculada por meio da equação $\mathrm{O}$, com base nos seus respectivos raios iônicos $\left(\mathrm{r}_{0}\right)$ [27] e adotando-se a permissividade elétrica do vácuo $\left(\varepsilon_{0}\right)$ igual a $8,854.10^{-12} \mathrm{C}^{2} / \mathrm{J} . \mathrm{m}$.

[Figure 13: Estimated values of the electronic polarizability $\left(\alpha_{\mathrm{e}}\right)$ of ionic species(cations and anions) for the ceramic compounds silicon carbide $\left(\mathrm{Si}^{4+}\right.$ and $\left.\mathrm{C}^{4-}\right)$, alumina $\left(\mathrm{Al}^{3+}\right.$ and $\left.\mathrm{O}^{2-}\right)$ and partially stabilized zirconia with yttria (PSZ) $\left(\mathrm{ZR}^{4+}, \mathrm{O}^{2-}\right.$ and $\left.\mathrm{Y}^{3+}\right)$, calculated by using equation 15 , based on the ionic radii $\left(r_{0}\right)$ [27] and assuming the electric permittivity of vacuum ( $\varepsilon_{0}$ ) equal to $\left.8.854 .10^{-12} \mathrm{C}^{2} / \mathrm{J} . \mathrm{m}.\right]$

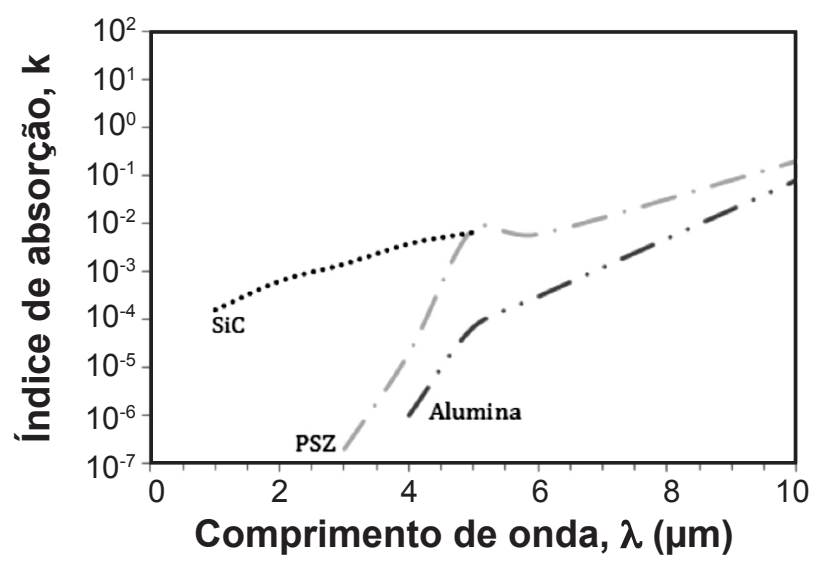

Figura 14: Índices de absorção $(\mathrm{k})$ da alumina $\left(\mathrm{Al}_{2} \mathrm{O}_{3}\right)$ [17], da zircônia parcialmente estabilizada com ítria (PSZ) [17], e do carbeto de silício (SiC) [28], obtido pela equação C, em função do comprimento de onda $(\lambda)$.

[Figure 14: Absorption index ( $k$ ) of alumina $\left(\mathrm{Al}_{2} \mathrm{O}_{3}\right)$ [17], zirconia partially stabilized with yttria (PSZ) [17], and silicon carbide (SiC) [28], calculated by equation $C$, as a function of wavelength $(\lambda)$.] 
comprimento de onda $(\lambda)$ de $4 \mu \mathrm{m}$ e também indica que os carbetos tendem a ser melhores absorvedores que os óxidos.

Compostos utilizados nas tintas de alta emissividade para veículos espaciais

Por meio dos conceitos apresentados anteriormente, as tintas de alta emissividade possuem aspectos relevantes que devem ser reforçados, são eles; a refletividade (R) e a absorção (A), e como estes estão relacionados com a emissividade $(\varepsilon)$ dos materiais. Para tanto, considerações teóricas sobre estes fenômenos foram abordados na parte inicial deste artigo. Quanto menor for à transmissão da radiação em questão, maior a parcela refletida ou absorvida. Além disso, a absorção e a emissão estão intimamente ligadas (equação Y), sendo que o aumento da primeira implica em uma maior emissividade. Deste modo, para tal aplicação são necessários compostos que não apenas sejam estáveis nas temperaturas de trabalho solicitadas, como também apresentem um índice de absorção $(\mathrm{k})$ elevado, pois quanto maior essa constante, maior a absorção (A) do material para um determinado comprimento de onda para uma dada espessura fixa. Isto se confirma ao se observar a evolução dos compostos utilizados pela NASA em suas tintas, os quais evoluíram do carbeto de silício para o dissiliceto de molibdênio e dissiliceto de tântalo, evidenciando a preferência por um aumento nos índices de absorção (k) como pode ser constatado na Fig. 15, na qual é apresentado o valor de $k$ em função do comprimento de onda $(\lambda)$ segundo os dados disponíveis na literatura.

A refletividade (R) também desempenha um papel importante neste contexto, como exposto na equação A. Vêse que a refletividade e a absorção são concorrentes, logo ao se reduzir a refletividade possibilita-se que o material absorva uma maior energia. Ainda, ao considerar a transmissão igual

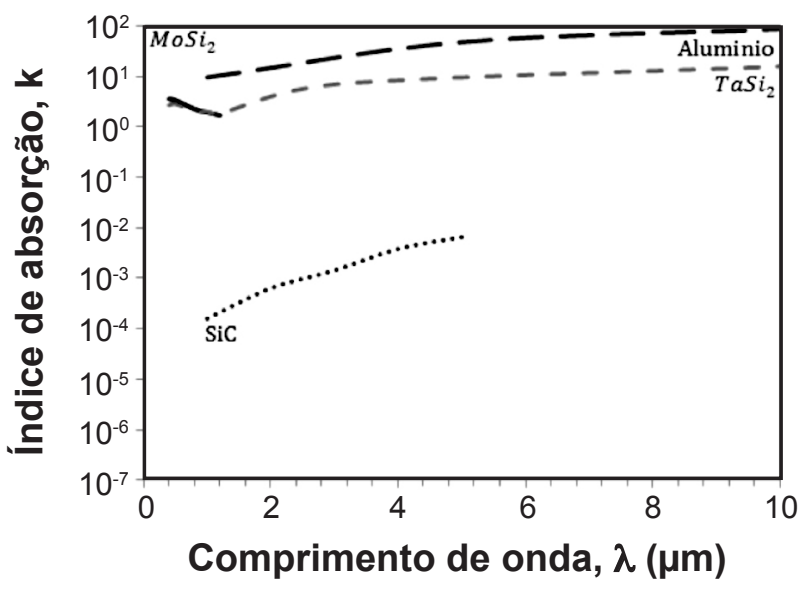

Figura 15: Índice de absorção $(\mathrm{k})$ do alumínio $\left(\mathrm{Al}_{2} \mathrm{O}_{3}\right)$, dos dissilicetos de molibdênio $\left(\mathrm{MoSi}_{2}\right)$ e tântalo $\left(\mathrm{TaSi}_{2}\right)$ [19], e do carbeto de silício ( $\mathrm{SiC}$ ) [28], obtido pela equação $\mathrm{C}$, em função do comprimento de onda $(\lambda)$.

[Figure 15: Absorption index ( $k$ ) of alumina $\left(\mathrm{Al}_{2} \mathrm{O}_{3}\right)$, molybdenum $\left(\mathrm{MoSi}_{2}\right)$ and tantalum $\left(\mathrm{TaSi}_{2}\right)$ [19] disilicides, and silicon carbide (SiC) [28],obtained by equation $\mathrm{C}$, as a function of wavelength $(\lambda)$.] a zero (ver equação A) chega-se na equação $\mathrm{AE}$, e como já mencionado, a absorção e a emissão estão diretamente relacionadas (equação $\mathrm{AD}$ ).

$$
(1-\mathrm{R})=\mathrm{A}
$$

Portanto, ao se observar a Fig. 16, nota-se que ambos dissilicetos $\left(\mathrm{MoSi}_{2}\right.$ e $\left.\mathrm{TaSi}_{2}\right)$ possuem uma refletividade inferior à do alumínio, o que possibilita uma maior penetração da radiação incidente no material. Assim, como seus índices de absorção (k) são praticamente iguais como se observa na Fig. 15, para uma mesma espessura (1) ambos absorvem a radiação incidente praticamente de forma igual

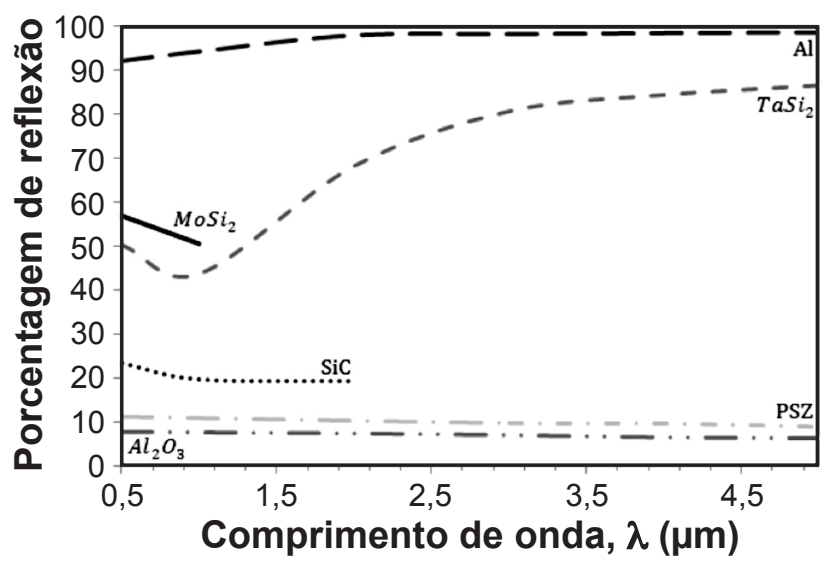

Figura 16: Refletividade de diversos materiais calculada pela equação $\mathrm{X}$ com base nos seus respectivos índices de refração (n) $[16,17,19,20]$ e absorção $(k)[16,17,19,28]$ para uma onda incidindo perpendicularmente à superfície.

[Figure 16: Reflectivity of various materials calculated by equation $X$, based on their refractive (n) $[16,17,19,20]$ and absorption $(k)$ $[16,17,19,28]$ indexes for a perpendicular incident wave on the surface.]

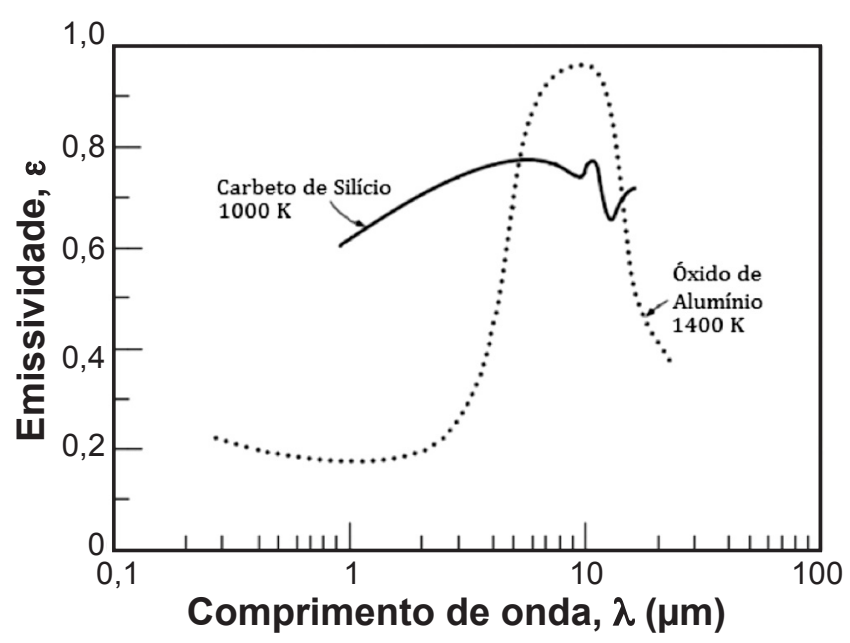

Figura 17: Emissividade $(\varepsilon)$ em função do comprimento de onda ( $\lambda$ ) para o carbeto de silício $(\mathrm{SiC})$ a $1000 \mathrm{~K}$ e para o óxido de alumínio $\left(\mathrm{Al}_{2} \mathrm{O}_{3}\right)$ a $1400 \mathrm{~K}$ [29].

[Figure 17: Emissivity ( $\varepsilon$ ) as a function of wavelength $(\lambda)$ for silicon carbide $(\mathrm{SiC})$ at $1000 \mathrm{~K}$ and for alumina $\left(\mathrm{Al}_{2} \mathrm{O}_{3}\right)$ at $1400 \mathrm{~K}$ [29].] 
(equação C). Por consequência, como a parcela de radiação refletida diminui para os dissilicetos, estes tem uma maior absorção em relação aos metais o que está de acordo com a equação AE. Portanto, a emissividade dos dissilicetos supera a dos metais, os quais geralmente possuem baixos valores devido à elevada refletividade que possuem.

Quando comparados às cerâmicas em geral, os dissilicetos também são superiores quanto a sua absorção, pois apesar da menor refletividade apresentada pelas cerâmicas (Fig. 16), os índices de absorção (k) destes materiais também são baixos (Fig. 1), ou seja, permitem a passagem da radiação através deles com facilidade, sem que esta seja necessariamente absorvida. Por meio da Fig. 12 torna-se possível justificar a elevada emissividade da terceira geração de tintas da NASA (Fig. 11), pois o valor de $\varepsilon$ para os picos de emissão nas temperaturas entre $500{ }^{\circ} \mathrm{C}$ e 2000 ${ }^{\circ} \mathrm{C}$, ou seja, para os comprimentos de onda $(\lambda)$ entre $3,8 \mu \mathrm{m}$ e 1,3 $\mu \mathrm{m}$ (equação AC), encontra-se na faixa entre 0,8 e 0,9. Logo a emissividade total $(\varepsilon)$ também se mantem entre estes valores (Fig. 9). Cabe lembrar que a queda de emissividade observada nos comprimentos de onda superiores tem pouca influência na emissividade total da tinta, pois estão distantes do pico de emissão na temperatura de trabalho do material. Adicionalmente, a queda de emissividade da primeira geração de tintas pode ser entendida por meio do comportamento do carbeto de silício ( $\mathrm{SiC}$ ), seu principal constituinte. Para este composto a emissividade diminui com a redução do comprimento de onda (Fig. 17). Portanto, a emissividade $(\varepsilon)$ da primeira geração de tintas decresce

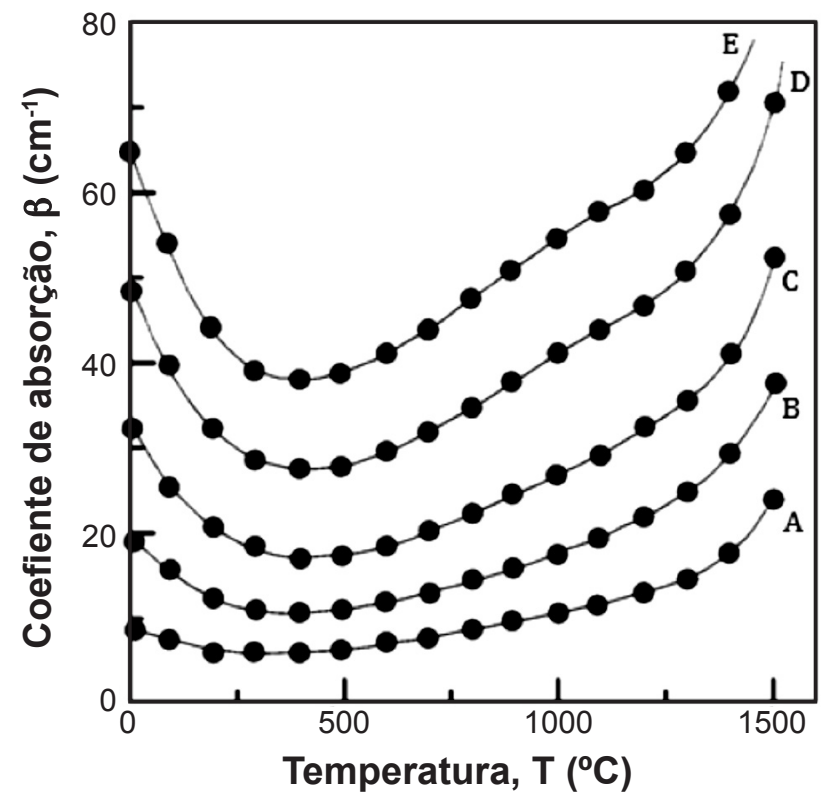

Figura 18: Comportamento do coeficiente de absorção $\left(\beta_{\mathrm{a}}\right)$ do carbeto de silício hexagonal $(6 \mathrm{H}-\mathrm{SiC})$ em função da temperatura $(\mathrm{T})$, para os comprimentos de onda $1,49 \mu \mathrm{m}, 2,00 \mu \mathrm{m}, 2,25 \mu \mathrm{m}, 3,03 \mu \mathrm{m}$ e 3,52 $\mu \mathrm{m}$, indicados respectivamente pelas letras de $\mathrm{A}$ a $\mathrm{E}$ [30].

[Figure 18: Absorption coefficient ( $\beta$ ) profile of hexagonal silicon carbide (6H-SiC) as a function of temperature (T), for the wavelengths $1.49 \mu \mathrm{m}, 2.00 \mu \mathrm{m}, 2.25 \mu \mathrm{m}, 3.03 \mu \mathrm{m}$ and $3.52 \mu \mathrm{m}$, shown by the letters A to E, respectively [30].] no intervalo de $980{ }^{\circ} \mathrm{C}$ a $1200{ }^{\circ} \mathrm{C}$, pois com o aumento da temperatura o comprimento de onda de pico de emissão diminui gradativamente, passando de 2,69 para 2,42 $\mu \mathrm{m}$ (equação $\mathrm{AC}$ ). Cabe salientar que a emissividade $(\varepsilon)$ do $\mathrm{SiC}$ para cada comprimento de onda permanece constante em relação à temperatura $(\mathrm{T})$ como apresentado na Fig. 18. A partir dos valores do coeficiente de absorção $\left(\beta_{\mathrm{a}}\right)$ do $\mathrm{SiC}$ em função da temperatura é possível calcular o índice de absorção (k) pela equação C. Logo, nota-se que a variação de $\beta_{\mathrm{a}}$, no máximo duplica o valor do índice de absorção (k). Entretanto, esta variação é insignificante quando considerada as ordens de grandeza deste índice e pode ser considerada nula. Este fato de independência da temperatura se deve aos índices de refração (n) e de absorção (k) serem funções fracas da temperatura.

\section{CONCLUSÃO}

Com base nos conceitos revisados ao longo deste artigo, verificou-se que o balanço entre a refletividade e a absorção dos materiais, define sua emissividade. Também fica claro que a absorção da radiação infravermelha ocorre de duas formas principais nos materiais, via transição eletrônica e por polarização, sendo a primeira claramente observada nos metais. Já nas cerâmicas predomina a polarização eletrônica e iônica, uma vez que nestes materiais a transição eletrônica só ocorre para comprimentos de onda menores, os quais por serem mais energéticos conseguem superar o intervalo entre bandas promovendo os elétrons presentes no material. Concluiu-se também que a última geração de tintas de alta emissividade da NASA destaca-se como sendo muito superior a primeira em decorrência das propriedades dos dissilicetos de molibdênio $\left(\mathrm{MoSi}_{2}\right)$ e de tântalo $\left(\mathrm{TaSi}_{2}\right)$ incorporados na sua fórmula, os quais são mais eficientes que os compostos cerâmicos anteriormente utilizados. Estes materiais possuem o aspecto predominante dos metais, ou seja, um elevado índice de absorção, sem, no entanto, terem a limitação da refletividade também muito alta. Sendo assim, a combinação destes fatores, alto índice de absorção e baixa refletividade, possibilita a estes intermetálicos uma maior absorção de energia e, consequentemente, uma maior emissividade tanto em relação aos metais quanto as cerâmicas, as quais apesar da baixa refletividade apresentam também baixo índice de absorção. Tal estudo e análises apresentadas adicionalmente fornecem a fundamentação adequada para o desenvolvimento de composições de tintas refratárias para aplicação em fornos industriais.

\section{REFERENCIAS}

[1] T. G. Evans, J. W. Olver, J. B. Dillard, J. A. Simmons, R. A. Churchward, "Thermal protective coating for ceramic surfaces", Patente US 6,921,431B2 (26/07/2005).

[2] D. A. Kourtides, R. A. Churchward, D. A. Lowe, "Protective coating for ceramic materials", Patente US 5,296,288 (22 March 1994).

[3] D. A. Stewart, D. B. Leiser, R. R. DiFiori, V. W. Katwala, 
"High efficiency tantalum-based ceramic composite structure", Patente US 7,767,305B1, 03 (10/2010).

[4] X. He, Y. Li, L. Wang, S. Zhang, "High emissivity coatings for high temperature application: progress and prospect", Thin Solid Films 517 (2009) 5120-5129.

[5] NASA, Columbia accident investigation board, Rept. 1, Washington D.C., EUA (2003) 49-78.

[6] E. Enofondos, W. F. Snyders, Rising producer prices in 1999 dominated by energy goods, Monthly Labor Rev. 123 (2000) 15-25.

[7] Buttonwood, Engine trouble, The Economist 397, 8705 (2010) 89.

[8] C. Crook, The new economy will be all about energy, National J. 37 (2005) 2255-2256.

[9] C. E. H. Jr., L. R. Champman, High emissivity coating, Patente 5,668,072 (16 Sept. 1997).

[10] G. A. Slack, Thermal conductivity of pure and impure silicon, silicon carbide, and diamond, J. Appl. Phys. 35 (1964) 3460-3466.

[11] G. A. Slack, Thermal conductivity of $\mathrm{MgO}, \mathrm{Al}_{2} \mathrm{O}_{3}$, $\mathrm{MgAl}_{2} \mathrm{O}_{4}$, and $\mathrm{Fe}_{3} \mathrm{O}_{4}$ crystals from 3 to $300 \mathrm{~K}$, Phys. Rev. 126 (1962) 427-441.

[12] M. F. Modest, "Radiative heat transfer", 2a. ed., Academic Press (2003) 4-60.

[13] M. Kirm, G. Zimmerer, Self-trapping and multiplication of electronic excitations in $\mathrm{Al}_{2} \mathrm{O}_{3}$ and $\mathrm{Al}_{2} \mathrm{O}_{3}: \mathrm{Sc}$ crystals, Phys. Rev. B 60 (1999) 502-510.

[14] ISO. ISO 21348: 2007(E), Space environment (natural and artificial) - Process for determining solar irradiances, $1^{\text {a }}$. ed. [S.1.]: ISO Copyright office (2007) 5-6 p.

[15] NASA. NASA's HEASARC: Tools. HEASARC

Energy Converter. Disponivel em: $<\mathrm{http} / /$ heasarc.nasa. gov/cgi-bin/Tools/energyconv/energyConv.pl>. Acesso em: fev. 2012.

[16] A. D. Rakic, Algorithm for the determination of intrinsic optical constants of metal films: application to aluminum, Appl. Optics 34 (1995) 4755-4767.

[17] J. Manara, M. Reidinger, S. Korder, M. ArduiniSchuster, J. Fricke, Development and characterization of low emitting ceramics, Int. J. Thermophys. 28 (2007) 1628-
1645.

[18] M. W. Barsoum, "Fundamentals of ceramics", $2^{\text {nd }}$ ed., IOP, Philadelphia, EUA (1997) 465-581.

[19] Sopralab, Disponivel em: <http://www.sopra-sa.com/ index2.php?goto=dl\&rub=4>. Acesso em: fev. 2012.

[20] Tropf, W. J.; Thomas, M. E.; Hopkins, J., Infrared refractive index and thermo-optic coefficient measurement at APL, Apl. Technical Digest 19 (1998) 293-298.

[21] D. O. Vivaldini, A. A. C. Mour'ao, V. R. Salvini, V. C. Pandolfelli, Revisão: Fundamentos e materiais para o projeto da microestrutura de isolantes térmicos refratários de alto desempenho, Cerâmica 60, 354 (2014) 297-307.

[22] N. S. Stoloff, S. T. Liu, S. C. Deevi, Emerging applications of intermetallics, Intermetallics 8 (2000) 13131320.

[23] D. M. Shah, D. Berczik, D. L. Alton, R. Hecht, Appraisal of other silicides as structural materials, Mater. Sci. Eng. A 155 (1991) 45-57.

[24] H. Zhang, X. Liu, Analysis of milling energy in synthesis and formation mechanisms of molybdenum disilicide by mechanical alloying, Int. J. Refractory Metals \& Hard Mater. 19, 2001. 203-208.

[25] J. J. Petrovic, R. E. Honnell, W. S. Gibbd, "Molybdenum disilicide alloy matrix composite", Patente US 5,069,841 (3 Oct. 1991).

[26] D. A. Bersztiss, R. R. Cerchiara, E. A. Gulbransen, F. S. Pettit, G. M. Meier, "Oxidation of $\mathrm{MoSi}_{2}$ and comparison with other silicide materials", Mater. Sci. Eng. A 155 (1992) 165-181.

[27] P. Atkins, L. Jones, "Princípios de química: questionando a vida moderna e o meio ambiente", $3^{\mathrm{a}}$. ed., Porto Alegre, RS, Bookman (2006) 840-849.

[28] G. B. Dubrovskii, E. I. Radovanova, Infrared impurity absorption in n-type silicon carbide, Phys. Status Solidi B 48 (1971) 875-879.

[29] Y. A. Cengel, "Heat transfer: a practical approach", $2^{\text {a }}$ ed., Mcgraw-Hill (2002) 580-581.

[30] R. Groth, E. Kauer, Absorption freier ladungsträger in a-SiC-kristallen, Physica Status Solidi B 1 (1961) 445-450. (Rec. 16/06/2012, Ac. 25/08/2012) 